\title{
BMJ Open Outpatient percutaneous native renal biopsy: safety profile in a large monocentric cohort
}

\author{
Dario Roccatello, ${ }^{1,2}$ Savino Sciascia, ${ }^{1,2}$ Daniela Rossi, ${ }^{1}$ Carla Naretto, ${ }^{1}$ \\ Mario Bazzan, ${ }^{3}$ Laura Solfietti, ${ }^{1}$ Simone Baldovino, ${ }^{1}$ Elisa Menegatti ${ }^{1}$
}

To cite: Roccatello D, Sciascia S, Rossi D, et al. Outpatient percutaneous native renal biopsy: safety profile in a large monocentric cohort. BMJ Open 2017;7:e015243. doi:10.1136/ bmjopen-2016-015243

- Prepublication history and additional material are available. To view these files, please visit the journal online (http://dx.doi. org/10.1136/bmjopen-2016015243).

DR and SS contributed equally.

Received 23 November 2016 Revised 9 May 2017 Accepted 10 May 2017

\section{(a) CrossMark}

${ }^{1}$ Department of Clinical and Biological Sciences, , Center of Research of Immunopathology and Rare Diseases-Coordinating Center of the Network for Rare Diseases of Piedmont and Aosta Valley, University of Turin, San Giovanni Hospital, Turin, Italy ${ }^{2}$ Nephrology and Dialysis Unit, S. Giovanni Bosco Hospital and University of Turin, Turin, Italy ${ }^{3}$ UOSD Hematology and Thrombosis Unit, S. Giovanni Bosco Hospital, Turin, Italy

Correspondence to Professor Dario Roccatello; dario.roccatello@unito.it

\section{ABSTRACT}

Objectives We aim to evaluate the safety of performing percutaneous native kidney biopsy (PKB) as an outpatient procedure (implying an observation period of 6 hours) compared with the traditional inpatient policy.

Design, setting, participants and measurements Group I, in whom PKB was performed in the outpatient department (2012-2016) and followed by 6 hours' observation period and then by regular outpatient visits and group II, in whom PKB was performed and followed by at least 1 day hospital admission. Group II included retrospectively retrieved patients who underwent PKB in our Institution between January 2000 and November 2012 as an inpatient procedure. All biopsies were performed by a single nephrologist following a structured protocol. Results 462 biopsies were reviewed, 210 (45.5\%) of patients were women and the mean age was $54.7 \pm 17.9$ years. One hundred and twenty-nine $(27.9 \%)$ of these biopsies were performed in outpatients. A total of 36 (7.8\%) of patients developed a complication, and of those, $9(1.9 \%)$ suffered for a major complication (arteriovenous fistula (six cases, $1.2 \%)$, ischaemic stroke $(2 ; 0.4 \%)$, thromboembolic pulmonary embolism $(1 ; 0.2 \%))$ and 27 (5.8\%) for minor(macroscopic haematuria (12 cases, $2.6 \%)$, haematomas on sonography not requiring intervention (15 cases, 3.2\%)). When comparing the complication rate between groups I and II, no statical difference was observed. When analysing together both groups, after multivariate analysis, serum creatinine $>3 \mathrm{mg} / \mathrm{dL}$ (OR 2.03, 95\% Cl 1.18 to 6.81) and known severe hypertension ( $\mathrm{OR} 2.01,95 \% \mathrm{Cl} 1.2$ to 4.7 ) were found to be independent risk factors for minor and major complications, respectively. Conversely, we found no association of risk with the number of biopsy passes, gender, age, diagnosis, presence of haematuria before the kidney biopsy nor the degree of proteinuria.

Conclusions Outpatient biopsy could be a valuable, safe and perhaps cost-effective method of obtaining diagnostic renal tissue in the majority of patients.

\section{INTRODUCTION}

Percutaneous biopsy of native kidneys is an important diagnostic tool for clinicians seeking a diagnosis for patients with kidney disease. The primary risks for percutaneous native kidney biopsy (PKB) range from mild complications such as postprocedural pain and gross
Strengths and limitations of this study

This is the largest reported cohort of percutaneous native kidney biopsies (PKB) performed in a single centre by a single experience nephrologist using automated devices and ultrasound guidance following a structured protocol.

- Our experience is not biased by heterogeneity in PKB approaches and level of expertise of the operator performing PKB.

- One limitation of our study is ambispective fashion of study design. Both prospective and retrospective data were studied.

haematuria to major complications such as large haematomas requiring blood transfusion, uncontrolled bleeding requiring embolisation or surgical nephrectomy and rarely death. ${ }^{1}$ The technique for obtaining tissue has evolved with the emergence of direct ultrasound guidance as the standard of care, dramatically improving procedural safety and diagnostic yield. ${ }^{2}$ While a number of centres worldwide require overnight inpatient observation (IO) following PKB, several studies have suggested the safety of the outpatient 'day surgery' (ODS) approach. ${ }^{23}$ However, to date, debate still exists on the appropriate observation time after PKB. In fact, despite some studies showing that discharging patients within 4-6hours after biopsy seems to be safe, ${ }^{4-6}$ Whittier and Korbet found that an observation period of less than 8 hours following biopsy missed $33 \%$ of complications. $^{7}$

We carried out a prospective observational study over a 5-year period of consecutive outpatient native renal biopsies to evaluate safety of ODS-PKB. Outcomes and the rate of complications after ODS-PKB were compared with IO-PKD performed in our Institution between January 2000 and November 2012. Besides, we aimed to identify preprocedure risk factors for complications (either minor or major) after a PKB. 


\section{MATERIALS AND METHODS}

\section{Patients selection}

For the purpose of this study, two groups of patients were considered: group I, in whom renal biopsy was performed in the outpatient department (2012-2016) and followed by 6 hours' observation period and then by regular outpatient visits and group II, in whom kidney biopsy was performed and followed by at least 1 day hospital admission.

Patients in group I were prospectively enrolled since January 2012, when we began performing renal biopsies as outpatient procedures in all consecutive patients using a standardised outpatient protocol (as provided in the supplementary material). A prospective computerised database was used to enter the data.

Group II included retrospectively retrieved patients who underwent PKB in our Institution between January 2000 and November 2012 as inpatient procedure. Patients whose data set was not fully available were excluded from our analysis (three cases).

PKB were performed when needed as part of good clinical practise for patients refereed to our department. Data collection was performed according to the local legislation of the institutional review board.

\section{Pre-ODS-PKB standardised procedures}

Patients are scheduled within 3 to 7 days from the day of call. Pre-ODS-PKD standardised procedures includes cell blood count $(\mathrm{CBC})$, renal function panel, coagulation profile (prothrombin time, partial thromboplastin, bleeding time, fibrinogen, PFA-100, platelet count) and ECG. Antiplatelets/anticoagulant therapies are screened and haematological evaluation is routinely requested in patients requiring therapy adjustment prior to biopsy.

\section{Real-time ultrasound-guided renal biopsy}

All biopsies were performed by a single nephrologist (DR) with the guidance of an expert radiologist who also performed an ultrasound examination of the kidney prior to PKB. PKB is performed following a structured protocol. In brief, the skin is prepped with antiseptic solution and draped in the customary fashion. A sterile cover is placed over the ultrasound probe and the kidney visualised. The skin and subcutaneous tissue are anaesthetised with lidocaine. The automated biopsy gun (needle 18 gauge, $15 \mathrm{~cm}$ ) was used. Under real-time ultrasound guidance, the biopsy needle gun is advanced. Once it is close to the renal capsule, the gun is fired with the patient holding his or her breath. The biopsy needle is retrieved, and the specimen placed in a media container and sent to surgical pathology. Three passes are performed per patient. As stated in the protocol, desmopressin acetate is routinely administered prior to PKB $(0.4$ microgr $/ \mathrm{Kg})$.

\section{Post-ODS-PKB standardised monitoring}

Patients are placed in a prone position on the bed for at least 2 hours. Patients received intravenous hydration and are observed for symptoms of urine retention.
Monitor-urine analysis for microscopic or macroscopic haematuria is routinely performed. Half-hourly measurements of pulse and blood pressure for 2 hours after PKD and then hourly until discharge are performed. Postbiopsy imaging was done in all the patients following the protocol. Additional imaging investigations, including additional sonography were performed when clinically indicated at the discretion of the attending physician.

\section{Minor or major complication definition}

Postbiopsy bleeding complications were categorised as either minor or major. Minor complications included gross haematuria and/or subcapsular perinephric haematoma $(<5 \mathrm{~cm}$ diameter $)$ that spontaneously resolved without the need for further intervention. Major complications were defined as those that required an intervention for resolution, either the transfusion of blood products or an invasive procedure (angiography, surgery), and those that might potentially led to acute renal obstruction or failure, septicaemia or death.

Subcapsular haematoma $>5 \mathrm{~cm}$ and/or those requiring intervention (despite the size) were considered major complications

The size of postbiopsy haematomas (surface area) was defined as the product of the longest and the shortest diameters on the two-dimensional sonographic pictures.

\section{Data analysis}

The prospective database included demographic and clinical features, laboratory values, biopsy complications and diagnostic or therapeutic procedures to manage haemorrhagic complications. In addition, the surgical pathology reports were used to ascertain the adequacy of renal tissue and pathological diagnosis. Univariate analysis was performed to assess the association between complications and risk factors using the Pearson, $\chi^{2}$ and Fisher exact tests. For univariate analysis, the following variables were considered in the model: number of biopsy passes, gender, age, diagnosis, kidney size at sonographic investigations, presence of haematuria before the kidney biopsy, the degree of proteinuria, haemoglobin level before the kidney biopsy, thrombocytopenia, severe arterial hypertension, serum creatinine level before the kidney biopsy, the use of antiplatelets, low molecular weight heparin (LMWH) and antihypertensive agents.

Multivariate logistic regression analysis was performed to identify significant independent factors adjusted for the potential confounding risk factors able to predict a complication; the results are expressed as OR with 95\% CI. The final multivariate logistic regression model includes the following variables: gender, age, diagnosis, the degree of proteinuria, haemoglobin level before the kidney biopsy, severe arterial hypertension, serum creatinine level before the kidney biopsy, the use of antiplatelet agents (as described in online Supplementary table 1s). The forward conditional techniques were used to find the final model. 
Table 1 Demographic, clinical and laboratory findings in groups I and II

\begin{tabular}{|c|c|c|c|c|}
\hline & 2000-2015 & 2000-2012 (inpatients) & 2012-2015 (outpatients) & p Value \\
\hline Biopsies & 462 & 333 & 129 & \\
\hline Passages, mean $\pm S D /$ median (range) & $2.6 \pm 0.7 / 3(1-5)$ & $2.9 \pm 0.6 / 3(1-5)$ & $3.1 \pm 0.6 / 3(2-5)$ & NS \\
\hline Glomeruli, mean $\pm \mathrm{SD} /$ median (range) & $19.9 \pm 11.3 / 18(6-71)$ & $21.6 \pm 12.4 / 20(1-71)$ & $23.9 \pm 12 / 23(3-58)$ & 0.01 \\
\hline Age, mean $\pm S D$ & $54.7 \pm 17.9$ & $56 \pm 19$ & $52 \pm 17.6$ & NS \\
\hline Female, n (\%) & $180(39)$ & $114(34)$ & $66(51)$ & NS \\
\hline $\begin{array}{l}\text { Admission duration (days), mean } \pm \mathrm{SD} / \\
\text { median (range) }\end{array}$ & - & $1.1 \pm 0.6 / 1(1-7)$ & - & \\
\hline $\mathrm{sCr}(\mathrm{mg} / \mathrm{dL})$, mean $\pm \mathrm{SD} /$ median (range) & $1.67 \pm 1.2 / 1.3(0.5-7)$ & $1.56 \pm 0.9 / 1.3(0.5-6)$ & $1.8 \pm 1.24 / 1.4(0.5-7)$ & NS \\
\hline $\mathrm{sCr}>3(\mathrm{mg} / \mathrm{dL}), \mathrm{n}(\%)$ & $124(26.8)$ & $89(29.6)$ & $35(27.1)$ & NS \\
\hline $\begin{array}{l}\text { Proteinuria ( } g / 24 \text { hours), mean } \pm S D / \\
\text { median (range) }\end{array}$ & $2.6 \pm 2.3 / 2(0.0-13)$ & $2.7 \pm 2.2 / 2(0.0-10)$ & $2.6 \pm 2.2 / 2(0.2-13)$ & NS \\
\hline Prebiopsy haematuria, n (\%) & $375(81.2)$ & $261(78.4)$ & $114(88.4)$ & 0.01 \\
\hline Resistant hypertension*, n (\%) & $110(23.9)$ & $93(27.9)$ & $17(13.2)$ & 0.008 \\
\hline Complications (any), n (\%) & $36(7.8)$ & $24(7.2)$ & $12(9.3)$ & NS \\
\hline Minor, n (\%) & $27(5.8)$ & $19(5.7)$ & $8(6.2)$ & NS \\
\hline Major, n (\%) & $9(1.9)$ & $5(1.5)$ & $4(3.1)$ & NS \\
\hline
\end{tabular}

*Mancia G, Fagard R, Narkiewicz K, et al. 2013 ESH/ESC Guidelines for the management of arterial hypertension: the task force for the management of arterial hypertension of the European Society of Hypertension (ESH) and of the European Society of Cardiology (ESC). $J$ Hypertens 2013;31:1281-357.

In order to analyse risk factors associated with time to complication, multivariate survival analysis was performed using the proportional hazards model (Cox model) in the prospective arm of the study. Risk factors included sex, diagnosis (categorised in primary glomerulopathy or systemic autoimmune condition), age $\geq 60$, kidney size $<8 \mathrm{~cm}$ at sonographic investigations, haematuria, nephrotic proteinuria, haemoglobin level $(<10 \mathrm{~g} /$ $\mathrm{dL}$ ), thrombocytopenia, severe arterial hypertension, serum creatinine $>3 \mathrm{mg} / \mathrm{dL}$, use of antiplatelets, LMWH and antihypertensive agents.

\section{RESULTS}

A total of 462 biopsies (group I and group II) were included in this study, $210(45.5 \%)$ of patients were women and the mean age was $54.7 \pm 17.9$ years. Table 1 summarised demographic, clinical and laboratory findings in the whole cohort.

Twenty-three per cent of biopsies were performed for the diagnostic workup of nephrotic range proteinuria, $16 \%$ for rapidly progressive renal insufficiency, $8 \%$ for acute kidney injury, $14 \%$ for a chronic kidney disease and the remaining $39 \%$ for non-nephrotic proteinuria and/ or haematuria.

A total of $36(7.8 \%)$ of patients developed a complication, and of those, $9(1.9 \%)$ suffered for a major complication (arteriovenous fistula (six cases, 1.2\%), ischaemic stroke $(2,0.4 \%)$, thromboembolic pulmonary embolism $(1,0.2 \%))$ and $27(5.8 \%)$ for minor (macroscopic haematuria (12 cases, 2.6\%), haematomas on sonography not requiring intervention (15 cases, $3.2 \%)$ ).

\section{Inpatients and outpatients}

One hundred and twenty-nine $(27.9 \%)$ of these biopsies were performed as outpatients and prospectively included. Data from 333 PKD performed as inpatients were retrospectively collected and analysed. Table 1 summarised demographic, clinical and laboratory findings, dividing patients in groups I and II.

Outpatients differed from inpatients with regard total glomeruli obtained (median=23 (1-71) vs median=20 $(3-58), \mathrm{p}=0.01)$, prevalence of prebiopsy haematuria $(78.4 \%$ vs $88.4 \%)$ and severe hypertension $(13.2 \%$ vs $27.9 \%$ ). When comparing the complication rate between groups I and II, no statical difference were observed (overall, 24 (7.2\%) complications in group I and 12 $(9.3 \%)$ in group II; $5(1.5 \%)$ and $4(3.1 \%)$ major, 19 $(5.5 \%)$ and $8(6.2 \%)$ minor complications, respectively in groups I and II).

\section{Assessment of potential predictors of postbiopsy complications}

When analysing together both groups, after multivariate analysis, serum creatinine $>3 \mathrm{mg} / \mathrm{dL}$ (OR 2.03, 95\% CI 1.18 to 6.81 ) and known severe hypertension (OR 2.01, $95 \%$ CI 1.2 to 4.7 ) were found to be independent risk factors for minor and major complications, respectively. Conversely, we found no association of risk with the number of biopsy passes, gender, age, diagnosis, kidney size at sonographic investigations, presence of haematuria before the kidney biopsy nor the degree of proteinuria. When focusing the survival prospective analysis only on group I, a similar trend was observed, but it failed to reach 
a statistical significance. Online Supplementary table 1s summarises the factors associated with the presence of complication in the univariate and multivariate models (see online Supplementary file 2).

\section{DISCUSSION}

The main finding in the present study is that a renal biopsy should be regarded as a safe procedure because it has a less than $2 \%$ overall rate of major complications. Importantly, when comparing the complication rate between groups I and II, no statical difference was observed, also when stratifying patients for major and minor complications (overall, $7.2 \%$ complication in group I and $9.3 \%$ in group II; $1.5 \%$ and $3.1 \%$ major, $5.5 \%$ and $6.2 \%$ minor complications, respectively, in groups I and II). Although the study was not randomised, it was performed prospectively with a proper follow-up of the patients.

These results are in line with Lin $e t a l^{8}$ who found that there is no difference in the rate of complications between patients who are admitted and those observed for a 6-hour period, the latest being acceptable. By contrast, Whittier and Korbet ${ }^{7}$ found that $42 \%$ of complications following native kidney biopsy manifested at $\leq 4$ hours, $67 \%$ at $\leq 8$ hours, $85 \%$ at $\leq 12$ hours and $89 \%$ at $\leq 24$ hours.

The main reason for overnight stay in the hospital is basically as a safety net in case there is a major complication. $^{9}$ The major complication, which one could encounter, is severe bleeding causing a large retroperitoneal haematoma. This complication can be catastrophic and should be addressed immediately by performing a selective renal arteriogram with embolisation of the bleeding arteriole, which will infarct a small portion of the kidney. This complication is in the order of $0 \%$ to $6 \%$ depending on the authors ${ }^{27-17}$; the reasons for these differences are not cleared but may be related to the technique used (blind vs ultrasound-guided biopsy), operator experience, gauge of the biopsy needle and the number of passes. We demonstrated lower frequency of haemorrhagic complications with real-time ultrasound-guided biopsies, as compared with blind biopsies. ${ }^{10}$

Some authors believe that patients are still at risk for type complication beyond the 8-hour observation postbiopsy; we hypothesise that under a controlled environment (see standardised protocol) and a proper technique (real-time ultrasound), we can minimise this risk and be able to have the renal biopsy performed as an outpatient procedure. In detail, Marwah and Korbet ${ }^{9}$ in their study accounted together all complications (minor and major), and in their cohort, only $42 \%$ of the patients had the biopsy performed with an automated gun, the rest were performed with a manual biopsy device and all biopsies were performed with 14-gauge needles and there was no report on how many passes were performed. They timed the major complications, which accounted for 24 out of a total of 394 biopsies (6.6\%) and reported that 19 of them were observed before the 8-hour mark.
Thus, only five major complications were captured after 8 hours of observation. Subsequently, when Whittier and Korbet ${ }^{7}$ re-evaluated the data and reported a series of 750 patients, in which they added the patients from the prior study, they concluded that less than 8 hours period of observation was not optimal and they reported that it could miss up to $33 \%$ of complications. Again, all complications (minor and major) were placed in the same category. Out of 750 biopsies, 45 had a major complication $(6.6 \%)$. Thirty of them were diagnosed before 8 hours of observation, the other 15 were diagnosed between 9 and 24 hours. On the contrary, there are at least four studies showing different results. Fraser and Fairley reported only minor complications in a series of 118 patients (only two patients). ${ }^{4}$ Oviasu and Ugdodaga $^{5}$ from Nigeria reported no complications in a series of 20 patients. Murphy and MacIsaac ${ }^{6}$ had similar data. Bairy et al reported on 178 outpatient renal biopsies and reported no major complications with only $13.2 \%$ of minor complications to include four patients with gross haematuria, 16 patients with small perinephric haematomas and 3 with both haematuria and haematoma. ${ }^{3}$ No interventions were needed, and only two patients stayed overnight.

The current study shows similar results.

It is worth noting that we observed three thrombotic events after PKD (two ischaemic strokes and one venous thromboembolism). As our protocol included the pre-PKD use of desmopressin, one could speculate a role of this agent in increasing the thrombotic risk. However, Manno et $a l^{18}$ when demonstrating in a double-blind randomised controlled clinical trial that prebiopsy desmopressin administration decreases the risk of bleeding and haematoma size in patients undergoing percutaneous kidney, they did not observe any episodes of thrombotic events in both desmopressin and control groups.

\section{Strengths and limitations}

This is the largest reported cohort of biopsies performed in a single centre by a single experience nephrologist using automated devices and ultrasound guidance following a structured protocol. Despite that the singlecentre cohort design may potentially limit the external validity of our findings, our experience is not biased by heterogeneity in PKB approaches and the level of expertise of the operator performing PKB.

One limitation of our study is ambispective fashion of study design. Both prospective and retrospective data were studied. These criteria were set a priori with the knowledge that cohort studies are prone to unpredictable bias and confounding by unknown factors and retrospective data analysis would only add to this risk. However, we used a multivariate analysis to ascertain the factors that contribute to postbiopsy complications, allowing for adjusting for potential known confounders, although unknown factors may not be accounted for. 
Finally, this study has also identified serum creatinine $>3 \mathrm{mg} / \mathrm{dL}$ and known severe hypertension as significant independent predictors of complications; however, when comparing groups I and II, a similar trend was observed, but it failed to reach a statistical significance. This may be due to the small number of major complications, especially major, observed in this study, and whether or not these same parameters are also important predictors of the major episodes occurring in ODS-PKD patients requiring intervention which is uncertain.

In conclusion, our study provides further evidence that it is safe to perform PKB as outpatient procedure after careful screening for risk of bleeding, using an automated needle-gun system under an ultrasound guide, following a standardised protocol. Therefore, same-day discharge with a 6-hour observation period seems a medically adequate procedure, and this represents a significant finding, since outpatient biopsies are economically advantageous.

Contributors DR performed the kidney biopsies. DR and SS designed the study, collected the data and drafted the manuscript. DR, CN and MB participated in the clinical evaluation, patients selection and data collection and critically reviewed the manuscript. LS, EM and SB performed the laboratory investigations and critically reviewed the manuscript. SB participated in the data analysis.

Competing interests None declared.

Patient consent Obtained.

Provenance and peer review Not commissioned; externally peer reviewed.

Data sharing statement The applied protocol for percutaneous kidney biopsies is attached and shared as supplementary material.

Open Access This is an Open Access article distributed in accordance with the Creative Commons Attribution Non Commercial (CC BY-NC 4.0) license, which permits others to distribute, remix, adapt, build upon this work non-commercially, and license their derivative works on different terms, provided the original work is properly cited and the use is non-commercial. See: http://creativecommons.org/ licenses/by-nc/4.0/

(C) Article author(s) (or their employer(s) unless otherwise stated in the text of the article) 2017. All rights reserved. No commercial use is permitted unless otherwise expressly granted.

\section{REFERENCES}

1. Parrish AE. Complications of percutaneous renal biopsy: a review of 37 years' experience. Clin Nephrol 1992;38:135-41 http://www.ncbi. nlm.nih.gov/pubmed/1395165

2. Maya ID, Maddela P, Barker J, et al. Percutaneous renal biopsy: comparison of blind and real-time ultrasound-guided technique. Semin Dial 2007;20:355-8.

3. Bairy M, Beleed K, Webb AT, et al. Safety of outpatient kidney biopsy: one center's experience with 178 native kidney biopsies. Am $J$ Kidney Dis 2008;52:631-2.

4. Fraser IR, Fairley KF. Renal biopsy as an outpatient procedure. Am J Kidney Dis 1995;25:876-8 http://www.ncbi.nlm.nih.gov/pubmed/ 7771483

5. Oviasu E, Ugbodaga P. Evaluation of percutaneous renal biopsy as a day case procedure: experience from Nigeria. $J$ Nephrol 1998;11:246-8 http://www.ncbi.nlm.nih.gov/pubmed/9831237

6. Murphy BF, Maclsaac A. Percutaneous renal biopsy as a day-patient procedure. Am J Kidney Dis 1989;14:77 http://www.ncbi.nlm.nih.gov/ pubmed/2741939

7. Whittier WL, Korbet SM. Timing of complications in percutaneous renal biopsy. J Am Soc Nephrol 2004;15:142-7.

8. Lin WC, Yang Y, Wen YK, et al. Outpatient versus inpatient renal biopsy: a retrospective study. Clin Nephrol 2006;66:17-24 http:// www.ncbi.nlm.nih.gov/pubmed/16878431

9. Marwah DS, Korbet SM. Timing of complications in percutaneous renal biopsy: what is the optimal period of observation? Am J Kidney Dis 1996;28:47-52.

10. Maya ID, Allon M. Percutaneous renal biopsy: outpatient observation without hospitalization is safe. Semin Dial 2009;22:458-61.

11. Medawar W, Green A, Campbell E, et al. Clinical and histopathologic findings in adults with the nephrotic syndrome. Ir J Med Sci 1990;159:137-40 http://www.ncbi.nlm.nih.gov/pubmed/2397982

12. Doyle AJ, Gregory MC, Terreros DA. Percutaneous native renal biopsy: comparison of a 1.2-mm spring-driven system with a traditional 2-mm hand-driven system. Am J Kidney Dis 1994;23:498-503.

13. Cozens NJ, Murchison JT, Allan PL, et al. Conventional $15 \mathrm{G}$ needle technique for renal biopsy compared with ultrasound-guided springloaded $18 \mathrm{G}$ needle biopsy. Br J Radiol 1992;65:594-7. 1.

14. Hergesell O, Felten $\mathrm{H}$, Andrassy K, et al. Safety of ultrasoundguided percutaneous renal biopsy-retrospective analysis of 1090 consecutive cases. Nephrol Dial Transplant 1998;13:975-7.

15. Marie I, Mouthon L. Therapy of polymyositis and dermatomyositis. Autoimmun Rev 2011;11:6-13.

16. Burstein DM, Korbet SM, Schwartz MM. The use of the automatic core biopsy system in percutaneous renal biopsies: a comparative study. Am J Kidney Dis 1993;22:545-52.

17. Burstein DM, Schwartz MM, Korbet SM. Percutaneous renal biopsy with the use of real-time ultrasound. Am J Nephrol 1991;11:195-200 http://www.ncbi.nlm.nih.gov/pubmed/1962666

18. Manno C, Bonifati C, Torres DD, et al. Desmopressin acetate in percutaneous ultrasound-guided kidney biopsy: a randomized controlled trial. Am J Kidney Dis 2011;57:850-5. 